Journal of Zhejiang University-SCIENCE B (Biomedicine \& Biotechnology)

ISSN 1673-1581 (Print); ISSN 1862-1783 (Online)

www.zju.edu.cn/jzus; www.springerlink.com

E-mail: jzus@zju.edu.cn

\title{
Editorial:
}

\section{Be careful! Avoiding duplication: a case study"}

Yue-hong (Helen) ZHANG ${ }^{\dagger}$, Xiao-yan JIA, Han-feng LIN, Xu-fei TAN

(Journals of Zhejiang University-SCIENCE $(A / B / C)$, Hangzhou 310027, China)

†E-mail: jzus@zju.edu.cn

doi: $10.1631 /$ jzus.B1300078

\section{Editors' bounden duty}

In recent years we have published many papers discussing how to avoid duplication (plagiarism) in scientific journals (Lin et al., 2009; 2011; Zhang, 2010a; 2010b; Zhang and Jia, 2012; 2013; Zhang and McIntosh, 2012; Jia and Zhang, 2013; Jia et al., 2013; Tan and Zhang, 2013). Indeed, to prevent plagiarism in scientific publishing, various detecting tools have been widely applied, such as CrossCheck, Turnitin, eBlast and AMLC (academic misconduct literature check) system of China National Knowledge Infrastructure (CNKI). In recent years, many journals have provided their explicit policies on this issue. For example, on Elsevier's website there are detailed instructions entitled "For Editors: Questions and Answers on Policies" (http://www.elsevier.com/ editors/perk/questions-and-answers), and also on our own journals' website (http://www.zju.edu.cn), there is "Instruction for Authors" that clearly states that we use "CrossCheck to fight against plagiarism and to ensure high ethical standards for all of the submitted papers". In short, as Meddings (2010) exclaims, "Credit where credit's due: plagiarism screening in scholarly publishing". Stopping plagiarism is the journal editors' responsibility (Zhang and McIntosh, 2012).

\section{Experience of using CrossCheck}

As editors of the Journals of Zhejiang University-

\footnotetext{
* Project supported by the Committee on Publication Ethics (COPE) (December, 2010) and the National Natural Science Foundation of China (No. 30824802)

(C) Zhejiang University and Springer-Verlag Berlin Heidelberg 2013
}

SCIENCE $(A / B / C)$, we have to point out that four years of experience tells us that CrossCheck is indeed an effective tool for detecting unoriginal content, enabling our editors to preserve our journal's integrity and the authors' copyright in our papers. However, CrossCheck is just a useful tool for the editor to find strings of similar text, but most instances of true plagiarism cannot be identified solely by these strings.

Fig. 1 shows our experience and basic referenced rules that we apply with regard to CrossCheck similarity indexes. First of all, we briefly introduce two concepts: (1) The overall similarity index (OSI) that means the total percentage of similarity between a submission and information existing in the CrossCheck/iThenticate databases selected as search targets; (2) The single match similarity index (SMSI) that means the percentage of similarity from a single source between the iThenticate database and the submitted document. If a paper has an OSI $>40 \%$ or an SMSI $>\sim 10 \%$, we usually reject it out of hand; if the OSI is between $\sim 25 \%$ and $\sim 40 \%$ or the SMSI is between $\sim 3 \%$ and $\sim 10 \%$, and if it appears in our editorial judgement that the ideas may have been plagiarized, the paper is usually returned for revision. Papers with an OSI $<\sim 25 \%$ and SMSI $<\sim 3 \%$, are handled on a case-by-case basis; in most cases, plagiarism is obscure, and we have to send the CrossCheck similarity report to the author and make sure about whether plagiarism is involved or not. If plagiarism is acknowledged, editors usually reject directly or return it for the author to re-write, based on the various conditions. As CrossCheck cannot detect all kinds of plagiarism and use of graphs etc., especially plagiarism of ideas, even a low similarity score does not mean there is no plagiarism. We always bear in mind that an anti-plagiarism tool cannot detect all problems, and more work in the checking process and use of peer reviewers' comments on originality and innovation are still the most important factors to ensure the quality of a paper. 


\section{What is the problem in method section of bioscience papers?}

In bioscience papers, besides the other scientific misconduct issues, replication of the method section is a common problem because duplication is always being detected in the section Materials and Methods. We editors often receive comments and queries from authors who think that it is a matter of course to copy their own published materials as opposed to copying those of others. How should editors handle such papers with similar content in the method section and how to guide authors in writing the method section without being accused of plagiarism? What is right? What is wrong? Here we studied an example to explain this problem.

\section{Case study}

In this case we asked the permission of the author and the reviewer to discuss this paper as an example of how to write the method section without too much repetition.

A manuscript submitted to Journal of Zhejiang University-SCIENCE B (Biomedicine \& Biotechnology) had gone through the peer review process; however, before making the final decision on publication, it is the journal's practice to run a CrossCheck. In this instance, the check revealed verbatim repetition of the description of the methodology used, from the author's own previous publications with only partial citation. A comparison of the similar sections is shown in Fig. 2a.

In the paper under study, the author used the same methods as described in her published papers to study a different topic. CrossCheck found more than 1000 words copied verbatim in the method section from four previously published papers by the same author. The similarity indices of these four papers were $15 \%, 6 \%, 3 \%$, and $1 \%$. The author was therefore asked to make revisions to avoid selfplagiarism.

In her first revision, the author completely replaced the description of the method with a citation, as shown in Fig. $2 b$. This avoids the problem of self-plagiarism, but the absence of any detail makes the method section look a little thin. One of the reviewers was asked for advice; his response was as follows:

"... If the method is exactly the same that [as] the original one, I suppose that may be correct to express in this way. But, usually there are minor modifications from previous methods. Indeed, in the manuscript I reviewed, authors said "Glucosinolates were extracted and analyzed as previously described with minor modifications (Yuan et al., 2009)". Besides, even if the method is the same that [as] a previous one, as a reader I think [it] is interesting to see some things in the paper. As [an] example, [does she] use the extraction method resin column or not? If yes, I am not interested in the method, if not then I can read the original method. Are glucosinolates by HPLC or MS, or GC? Depending on the method I may be interested in the original paper. So, I think that could be correct, but I don't like it and I think [it] is better if more information appears in Mat \& Meth. I hope that this thought can help you to take a decision."

Accordingly, the author was asked to make a second revision, providing proper citations and a clear but concise statement of the materials and methods used in each step, without too much surplus; this was satisfactorily done. Fig. $2 \mathrm{c}$ shows part of the method section. From such communication, the author showed that she would pay more attention to the method section to avoid repetition from now on.

From this case study, maybe authors will give enough information to avoid being accused of duplication in this section.

\section{Suggestions for author(s) reference}

In our current study (Zhang and Jia, 2012), we did a survey of the practice of leading journals, which shows various approaches to avoiding the verbatim repetition of previously published descriptions of methods. When the method is identical to that described in a previously published article (whether by the same or different authors), it is preferable to cite the previous article; if wording is repeated verbatim, it must always be credited to the original publication. Alternatively, a phrase such as "by standard method" or "as previously described" may be used, as in The Lancet etc. However, if the precise description of the method is especially important for understanding the 


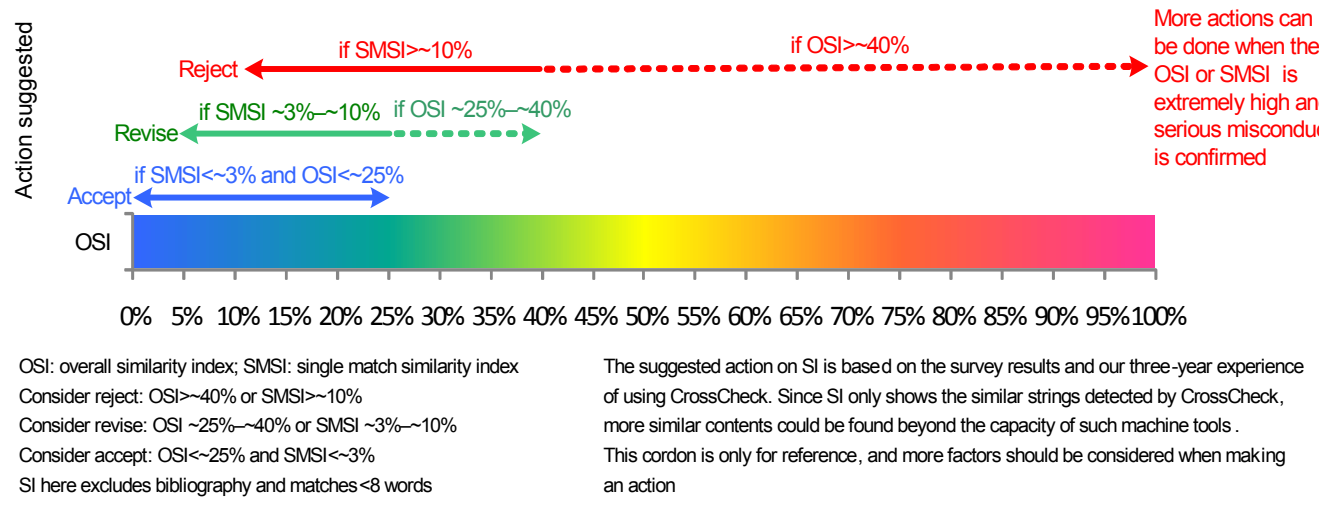

Fig. 1 Similarity score cordon of Journals of Zhejiang University-SCIENCE $(A / B / C)$ for CrossCheck user reference

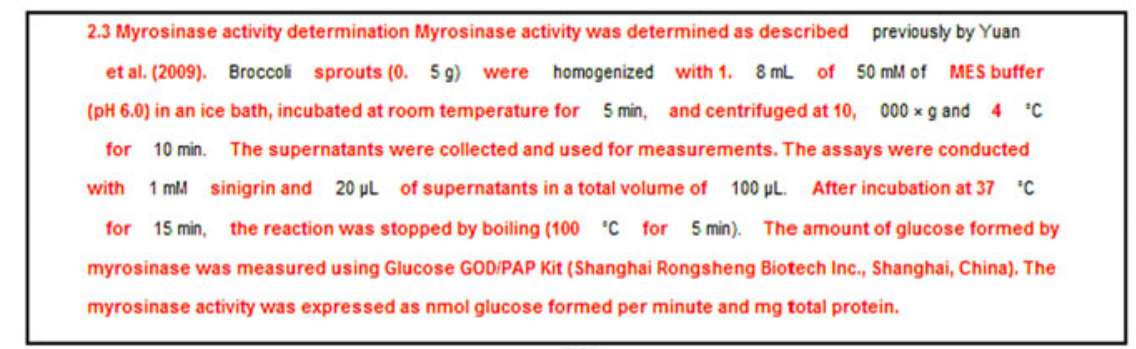

(a)

\author{
2.2 Glucosinolate assay \\ Glucosinolates were extracted and analyzed as previously described (Yu an et al., 2009). \\ 2.3 Myrosinase activity determination \\ Myrosinase activity was determined as described previously by Yuan et al. (2009). \\ 2.4 Sulforaphane measurement \\ Sulforaphane content was determined as described previously by Guo, Yuan \& Wang(2011).
}

(b)

\title{
2.2 Glucosinolate assay
}

Glucosinolates were extracted and analyzed as previously described with minor modifications (Yuan et al., 2009). $500 \mathrm{mg}$ sprouts were boiled in $3 \mathrm{~mL}$ water for two times respectively, and $1 \mathrm{~mL}$ of the combined aqueous extract was applied to a DEAE-Sephadex A-25 (35 mg) column (GE Healthcare, Piscataway, NJ). The desulphoglucosinolates were obtained according to the procedure of Yuan et al. (2009). Then the extraction was analyzed by high performance liquid chromatography (HPLC). The glucosinolate concentration was expressed as $\mu \mathrm{mol} / \mathrm{g}$ fresh weight of broccoli sprouts.

2.3 Myrosinase activity determination

Myrosinase activity was determined using spectrophotometry as described previously by Yuan et al. (2009). $500 \mathrm{mg}$ broccoli sprouts were ground with $1.8 \mathrm{~mL}$ of $50 \mathrm{mM} \mathrm{MES} \mathrm{buffer}(\mathrm{pH} 6.0)$ to homogenate at $0^{\circ} \mathrm{C}$, and then incubated at $25^{\circ} \mathrm{C}$ for $5 \mathrm{~min}$. After centrifuging at $10,000 \mathrm{rpm}$ and $4{ }^{\circ} \mathrm{C}$ for $10 \mathrm{~min}$, the supernatants were collected for measurements. The assay were followed the procedure of Yuan et al. (2009). The myrosinase activity was expressed as nmol glucose formed per minute and mg total protein.

(c)

Fig. 2 A case study of how to write the method section without too much repetition

(a) CrossCheck report highlighting areas of similarity to the author's previous publications; (b) The first revision of the method section, with citation only; (c) The second revision of the method section based on the suggestion of the reviewer, with rewording (including more details) and citation 
article, the attachment of supplemental material may be preferable, as in Science (Fig. 3). Only if the methods used are totally novel is it necessary to include a detailed description.

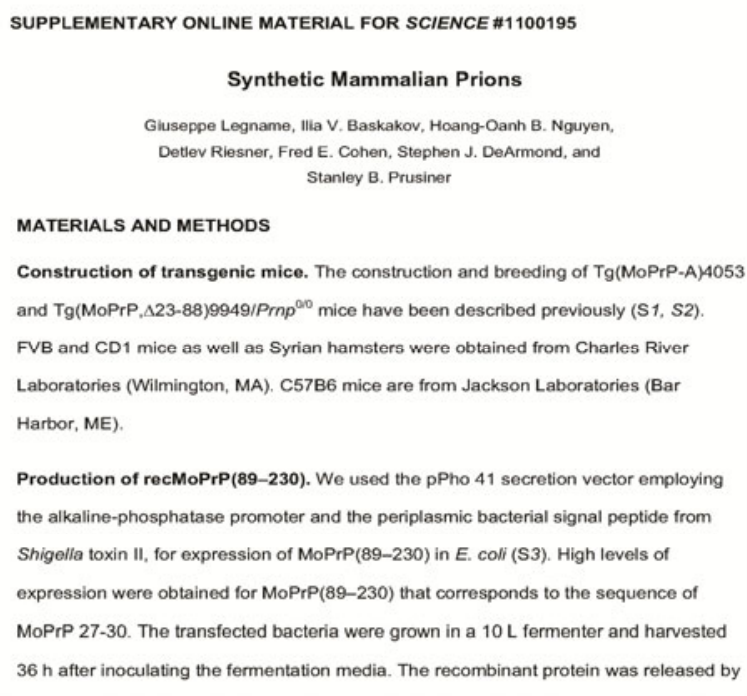

Fig. 3 Attachment sample from Science http://www.sciencemag.org/content/305/5684/673

Moreover, we also quote the useful words of a bio-scientist (Dr. McCanless) who suggested as follows:

1. If using kits and supplier is referenced, then this is enough due to most suppliers providing manuals.

2. If using traditional/homemade methods include following statement: "based on methods previously described" and include reference(s) as these procedures have all been documented in early chemistry/biochemistry journals.....this is how we know how to do them.

3 . If the procedure is novel or incorporates a novel step to a known method provide details (if the entire procedure is novel then a separate publication based on the method would be justified; if minor alterations are performed then state "based off [on] previously detailed protocols with the exception of..." Provide a reference for basic protocol and detail and innovative changes that were required to complete your experiment).

We think the three suggestions would be very useful for the author(s) reference in the biomedicine and biotechnology areas.

\section{References}

Jia, X.Y., Zhang, Y.H., 2013. Problem of duplication in the method section in bioscience papers: Part 2. China Publishing Journal, 309:6-8 (in Chinese).

Jia, X.Y., Tan, X.F., Zhang, Y.H., 2013. Replication of the method section in biosciences papers: is it plagiarism? Scientometrics, in press.

Lin, H.F., Zhang, X.X., Zhai, Z.Y., Wu, X.F., Zhang, Y.H., 2009. Using CrossCheck to protect original scientific research writing against academic misconducts. Chinese Journal of Scientific and Technical Periodicals, 20(4): 580-584 (in Chinese).

Lin, H.F., Jia, X.Y., Zhang, Y.H., Zhang, C.J., Jin, M.Q., Zhang, X.X., Zhai, Z.Z., 2011. Guarantee academic originality: duty of journal editors-workflow and analysis with CrossCheck of JZUS $(A / B / C)$. Chinese Journal of Scientific and Technical Periodicals, 22(3):328-333 (in Chinese).

Meddings, K., 2010. Credit where credit's due: plagiarism screening in scholarly publishing. Learned Publishing, 23(1):5-8. [doi:10.1087/20100102]

Tan, X.F., Zhang, Y.H., 2013. Problem of duplication in the method section in bioscience papers: Part 1. China Publishing Journal, 309:3-5 (in Chinese).

Zhang, Y.H., 2010a. Chinese journal finds $31 \%$ of submissions plagiarized. Nature, 467(7312):153. [doi:10.1038/467153d]

Zhang, Y.H., 2010b. CrossCheck: an effective tool for detecting plagiarism. Learned Publishing, 23(1):9-14. [doi:10.1087/20100103]

Zhang, Y.H., Jia, X.Y., 2012. A survey on the use of CrossCheck for detecting plagiarism in journal articles. Learned Publishing, 25(4):292-307. [doi:10.1087/2012 0408]

Zhang, Y.H., McIntosh, I., 2012. How to stop plagiarism: blacklist repeat offenders? Nature, 481:22. [doi:10.1038/ 481021a]

Zhang, Y.H., Jia, X.Y., 2013. Republication of conference papers in journals? Learned Publishing, in press. 